\title{
Motor performance and back pain in children and adolescents: a systematic review and meta-analysis protocol
}

\author{
Matias Noll ${ }^{1,2,3^{*}}$ (D) Niels Wedderkopp ${ }^{3,4^{*}}$ (D), Carolina Rodrigues Mendonça ${ }^{2}$ (D) and Per Kjaer ${ }^{3,3^{*}}$ (D)
}

\begin{abstract}
Background: The relationship between motor performance and back pain in children and adolescents remains unclear. This article describes the protocol for a systematic review to summarize the knowledge about the association between motor performance, such as agility, flexibility, balance, strength, muscle endurance, and cardiorespiratory fitness, and back pain. Thus, our aim is to identify the influence of motor performance on back pain among children and adolescents.
\end{abstract}

Methods: Two independent researchers will search MEDLINE, Scopus, Embase, SPORTDiscus, and CINAHL databases, with no period or language restrictions. We will include cross-sectional, cohort, case-control, and controlled clinical trial studies based on the following criteria: (a) participants from 6 to 19 years of age, (b) assessment of motor performance, (c) assessment of back pain, and (d) report measures of associations between motor performance and back pain. Study quality and risk of bias will be assessed using an adapted version of the Downs and Black instrument. Grading of Recommendations, Assessment, Development, and Evaluations will be used to assess the strength of the body of evidence. Meta-analyses of association measures will be performed for each type of motor performance, separately for different study types. The results will be reported using forest to show the pooled effect of findings and funnel plots to assess precision of the data. If studies are not homogeneous, results from the meta-analyses will not be reported. Associations will then be synthesized descriptively using a pragmatic approach.

Discussion: This systematic review will provide critical insights into the association between motor performance and back pain among children and adolescents; this information may help support clinical practice guidelines as well as public health programs.

Ethics and dissemination: Protocol was written according to the Preferred Reporting Items for Systematic reviews and Meta-Analyses (PRISMA).

Systematic review registration: PROSPERO CRD42020178496

Keywords: Back pain, Pain, Teenagers, Psychomotor performance, Protocol, Systematic review

\footnotetext{
*Correspondence: matias.noll@ifgoiano.edu.br; nwedderkopp@health.sdu.dk;

pkjaer@health.sdu.dk

'Instituto Federal Goiano, Ceres, Brazil

${ }^{3}$ Department of Sports Science and Clinical Biomechanics, University of

Southern Denmark, Campusvej 55, Odense, Denmark

Full list of author information is available at the end of the article
}

(c) The Author(s). 2020 Open Access This article is licensed under a Creative Commons Attribution 4.0 International License, which permits use, sharing, adaptation, distribution and reproduction in any medium or format, as long as you give appropriate credit to the original author(s) and the source, provide a link to the Creative Commons licence, and indicate if changes were made. The images or other third party material in this article are included in the article's Creative Commons licence, unless indicated otherwise in a credit line to the material. If material is not included in the article's Creative Commons licence and your intended use is not permitted by statutory regulation or exceeds the permitted use, you will need to obtain permission directly from the copyright holder. To view a copy of this licence, visit http://creativecommons.org/licenses/by/4.0/ The Creative Commons Public Domain Dedication waiver (http://creativecommons.org/publicdomain/zero/1.0/) applies to the data made available in this article, unless otherwise stated in a credit line to the data. 


\section{Background}

Back pain (BP), defined as "pain within the cervical, thoracic and/or lumbar areas" [1-3], is a significant public health problem [1-7]. Recent studies [8-10] suggest that the prevalence of $\mathrm{BP}$, including acute, chronic, specific, and non-specific conditions, has been increasing among adolescents; it can cause a range of functional limitations that may persist into adulthood [11-13]. Therefore, the causes of BP among adolescents should be investigated [14]. Studies have shown that income level [9], sex [15], psychological and social factors [16], sedentary behavior [17], sleep problems [18], and cardiovascular risks [19] may be associated with BP among children and adolescents. Several studies have focused on the relationship between physical activity [20] or sports variables (e.g., the type of sport and frequency and intensity of exercise) [21-25] and BP.

The relationship between motor performance and BP remains unclear. Motor performance components are essential to psychological and social development in children and adolescents [26] and have been recognized as an important determinant of current and future health status [27, 28]. Recent reviews [1, 2, 29] evaluating potential risk factors for $\mathrm{BP}$ pain in children and young adults reported that there were few studies on components of motor performance, including agility, strength in the lower extremity, hand grip strength, and cardiorespiratory fitness, and that these relationships are unclear. Motor performance is an important health parameter that is positively associated with physical activity [30]; however, it may also be related to injuries [31].

Pain during adolescence is an important predictor of pain in adulthood [32]. Therefore, an adequate assessment in children and adolescents is fundamental to help researchers and health professionals better understand BP and associated risk factors to improve health promotion programs and interventions. To the best of our knowledge, there is a lack of understanding about the relation between the levels of motor performance, such as agility, flexibility, balance, strength, muscle endurance, and cardiorespiratory fitness and BP, as well as the particularities for sex and age groups. Thus, the present systematic review will focus on the following research question: "How could different levels of motor performance influence BP in young people?"

Our study aims to identify the influence of motor performance on BP in children and adolescents and to investigate the differences between sexes and age groups. Our main hypothesis is that higher levels of motor performance are related to reduced BP. Understanding these factors may enable health professionals to perform more targeted and effective health promotion interventions.

\section{Methods}

\section{Protocol and registration}

This systematic review will be conducted according to the Preferred Reporting Items for Systematic Reviews and Meta-analyses (PRISMA) guidelines [33] for the identification, screening, eligibility, and inclusion (Additional file 1). Our review follows the Population, Exposure, Comparator, and Outcome (PECO) structure, which is explained in the recommended notification items for systematic reviews [34]. Accordingly, "P" represents children and adolescents, "E" represents "motor performance," "C" represents "sex and age group," and "O" represents "BP variables."

The current protocol is registered in the International Prospective Register of Systematic Reviews (PROSPERO) [35] (Registration number CRD42020178496). Any amendments made to this protocol when conducting the study will be reported in PROSPERO and in the final manuscript.

\section{Search strategy and eligibility criteria}

In fall 2020, one researcher (MN) will search the MEDL INE, Scopus, Embase, SPORTDiscus, and CINAHL databases, with no period and language restriction. The detailed search strategy is presented in Table 1. Disagreement with respect to the searches will be discussed and resolved. In addition, search strategy will be supplemented by (a) citation tracking in the reference list of the included studies and relevant systematic reviews and (b) via Google Scholar searches.

We will include cross-sectional, cohort, case-control, and controlled clinical trial (data from control groups) studies. The inclusion criteria will be (a) participants aged 6 to 19, (b) assessment of motor performance, (c) assessment of BP, and (d) report measures of associations between motor performance and BP, or data that makes it possible to estimate associations.

"Motor performance" will be defined as "an observable action or behavior that can be measured (rated) by an observer" [36] which include the evaluation of agility, flexibility, balance, strength, muscle endurance, and cardiorespiratory fitness. BP is defined as "pain within the cervical, thoracic and/or lumbar areas including acute, chronic, specific, and non-specific conditions" [1-3].

\section{Table 1 Search strategy}

\begin{tabular}{l}
\hline = "back pain" OR backache OR backaches OR "back ache" OR "back \\
aches" OR "spinal pain" OR "spine pain" OR "neck pain" OR "neck ache" \\
OR "neck aches" OR "cervical pain" OR lumbago OR "lumbar pain" \\
2 = "Motor performance" OR "Motor performances" OR "fitness \\
performance" OR "fitness performances" OR "fitness assessment" OR \\
"movement assessment" OR "motor assessment" OR "psychomotor \\
performance" OR "psychomotor performances" OR "motor fitness" OR \\
Strength OR "abdominal muscular endurance" OR Flexibility OR Balance \\
OR Agility OR Jump OR "cardiorespiratory fitness" OR "cardiorespiratory \\
performance" OR "aerobic endurance" OR "anaerobic endurance" OR \\
"aerobic fitness" OR "anaerobic fitness" \\
3 = child OR children OR adolescent OR adolescents OR minors OR \\
youth OR youths OR juvenile OR teen OR teens OR teenager OR \\
teenagers OR childhood OR adolescence OR schoolchild OR "school \\
child" OR schoolchildren OR "school children" OR young OR youngs \\
$4=1$ AND 2 AND 3
\end{tabular}


The exclusion criteria will be as follows: (a) studies with incomplete data or review articles, opinion articles, and case reports; (b) non-quantitative measures in the assessment of motor performance; (c) specific populations that included athletes, hospitalized adolescents, diseased people, disabled people, amputees, or pregnant and lactating women; (d) mixing of athletes with nonathletes, unless the data were reported separately or could be calculated from the data provided; and (e) mixing of children and adolescents with other group ages, unless the children's and adolescents' data were reported separately or could be calculated from the data provided.

\section{Training of the reviewers}

The authors who will participate in eligibility assessments are being trained regarding the study inclusion/exclusion criteria and will complete practice eligibility assessments on 50 test abstracts before starting to code articles. The authors also will train in performing risk of bias instruments on five not-included articles, as well as standardized analyses using Mendeley and Rayyan software.

\section{Review process}

After executing the search strategy, articles will be collated, and duplicates will be removed using the Mendeley Software. Then, two reviewers (MN and CRM) will independently screen the titles and abstracts of all articles identified in the literature search for inclusion. Disagreement regarding inclusion will be discussed and resolved by a third reviewer (PK or NW). The screening process will be performed for both reviewers using Rayyan Software [37]. Inter-rater reliability for individual component ratings will be determined by calculating the percentage of agreement and Cohen's Kappa coefficient [38]. The remaining articles will be read in full and evaluated to determine their eligibility based on the inclusion and exclusion criteria. Finally, the eligible articles will be included in the systematic review. In addition, the reference lists of included articles will be searched to identify additional studies missed by database searches. The flow chart for this systematic review is shown in Fig. 1.

\section{Data extraction and quality assessment}

From the selected articles, the following data will be extracted: authors, publication year, ethical approval, conflict of interest, sample size, sex, age, location/country, study design, main and secondary outcomes, instruments for measurement, major findings, and association values. Regarding $\mathrm{BP}$, we will record the pain definition, time since onset, prevalence, frequency, intensity, and site(s). These characteristics of the articles included in the systematic review will be presented in Additional file 2 and Tables 2 and 3. If during the process, needs to include more information arise, more columns will be included in these Tables.

Risk of bias will be assessed using the 27-item Downs and Black instrument [39]. For observational studies, not all items on the Downs and Black checklist are generally applicable; therefore, a subset of 19 questions (questions

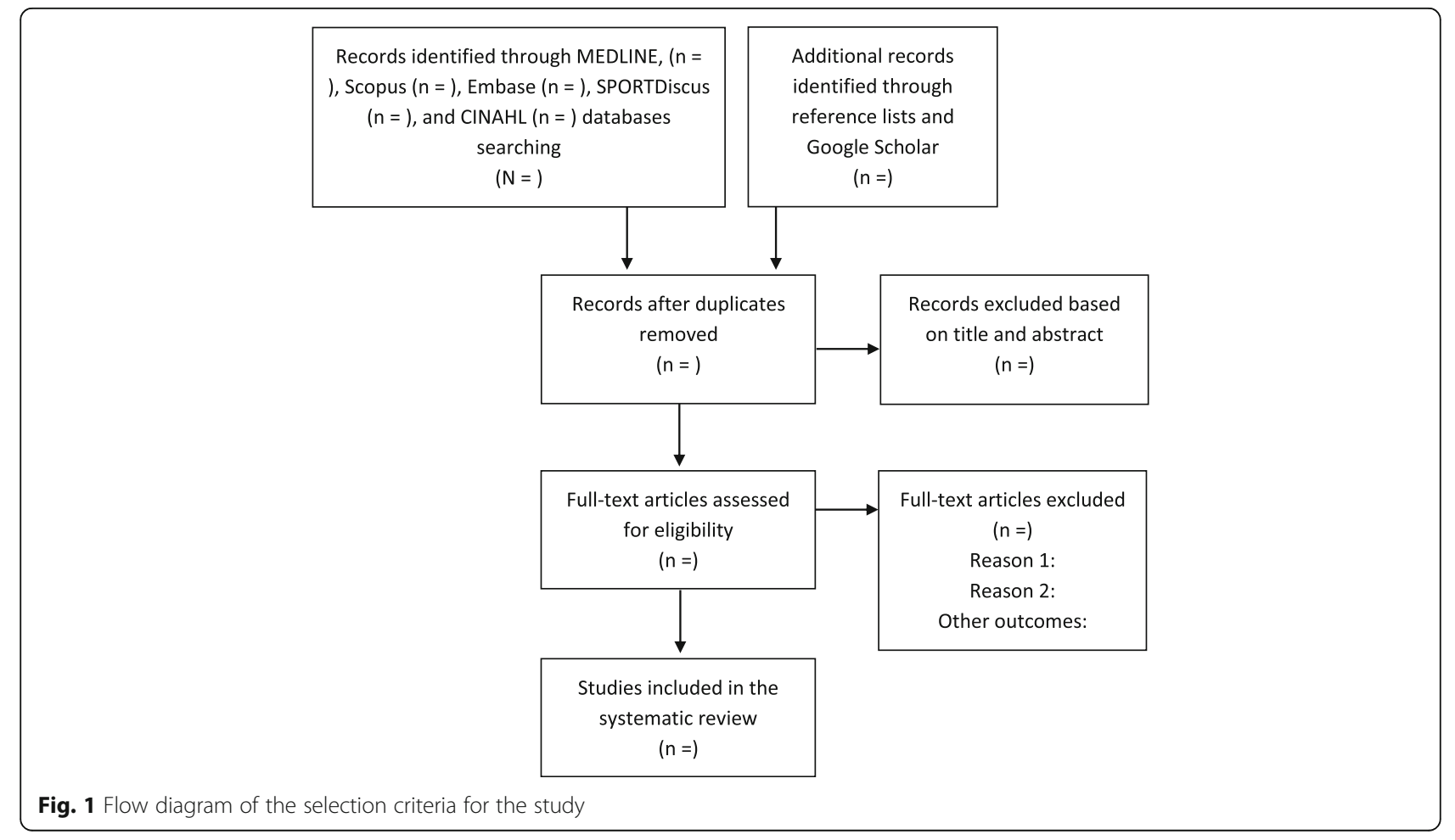


Table 2 Summary of the articles included in the systematic review

\begin{tabular}{l}
\hline Characteristics \\
\hline Publication year \\
Prior to 1990 \\
1991-2000 \\
2001-2010 \\
$2011-2020$ \\
Study design \\
Cross-sectional \\
Longitudinal \\
Intervention \\
Region/country \\
Region A \\
Region B \\
Region C \\
Region D \\
Region E \\
Region F \\
Sex \\
Male sex only \\
Female sex only \\
Mixed \\
Sample size \\
Outo0 \\
Outcooo performance variables \\
Outcome C \\
\end{tabular}

$1-3,5-7,9-12,15-18,20,21,25-27)$ will be used. A summary quality score (0-19 points) for each manuscript will be calculated by expressing the number of compliant items as a percentage of the total. Score above $70 \%$ will be used to define low risk of bias [39].

The Grading of Recommendations, Assessment, Development, and Evaluations (GRADE) evidence system will be used to assess the quality of evidence [40]. For each study, quality of evidence is ultimately assigned one of four grades: high quality, moderate quality, low quality, or very low quality [41] (Additional file 2).

We will also report whether the authors of the included studies stated potential conflicts of interest and gave information on ethical approval [42]. Data will be extracted and assessed by two independent reviewers ( $\mathrm{MN}$ and CRM), and disagreements will be resolved by a third reviewer (PK or NW). When relevant data are not available in the manuscript, a researcher (MN) will contact the authors directly to fill these gaps.

Our systematic review is focused on the analysis of published articles (secondary data), and it does not require ethics approval. After the conclusion, the authors will submit the systematic review for publication in a peer-reviewed journal.

\section{Evidence synthesis and statistical analyses}

We will present an overview of the available studies, describe the prevalence of the various types of BP, and report motor performance indicators in participants with and without BP. Absolute differences in motor performance and appropriate measures of association (relative risks, odds ratios, or diagnostic measures) will be compared.

Regarding these data, we plan to perform a metaanalysis if there are sufficient studies with low risk of bias $[39,43]$. Firstly, we will analyze the mean difference including 95\% confidence intervals in motor performance levels among children and adolescents with BP, considering the differences between girls and boys, and age groups, by either adjusting for or stratifying by sex and age groups. This will be reported separately for cross-sectional and longitudinal studies. Secondly, we will pool findings into random-effects meta-analysis and report these results using forest to show the pooled effect of findings and funnel plots to assess precision of the data [43]. Thirdly, the percentage of variability attributable to heterogeneity will be estimated using the $I^{2}$ test. $I^{2}$ values of $<40,40-60,60-90$, and $>90 \%$ correspond, respectively, to "not important," "moderate," "substantial," and "considerable" levels of heterogeneity as described by the Cochrane collaboration [43]. Metaanalysis will be performed in the STATA 16.0 Software (Stata Corporation, College Station, TX, EUA) at 95\% confidence interval. Publication bias will be assessed by visual inspection of a funnel chart. When interpreting the pooled estimates from each meta-analysis, the GRADE evaluation of each included study will be used to rate the overall quality of evidences.

Table 3 Quantitative characteristics of the articles included in the systematic review and results of the association

\begin{tabular}{|c|c|c|c|c|c|c|c|c|}
\hline $\begin{array}{l}\text { Author/ } \\
\text { year }\end{array}$ & $\begin{array}{l}\text { Design/ } \\
\text { country }\end{array}$ & $\begin{array}{l}\text { Subjects, N (\% } \\
\text { male) }\end{array}$ & $\begin{array}{l}\text { Age, (years/ } \\
\text { range) }\end{array}$ & $\begin{array}{l}\text { Motor } \\
\text { performance test }\end{array}$ & $\begin{array}{l}\text { Back pain } \\
\text { definition }\end{array}$ & $\begin{array}{l}\text { Region pain/ } \\
\text { period }\end{array}$ & $\begin{array}{l}\text { Pain } \\
\text { prevalence }\end{array}$ & $\begin{array}{l}\text { Main result (motor } \\
\text { performance } \times \text { pain) }\end{array}$ \\
\hline & & . & . & & . & . & . & . \\
\hline
\end{tabular}


We will present one meta-analysis for each motor performance test (agility, flexibility, balance, strength, muscle endurance, and cardiorespiratory fitness) as well as separately for cross-sectional and longitudinal studies. If metaanalyses are not appropriate, data will be synthesized descriptively using the forest plots and a pragmatic descriptive approach.

\section{Discussion}

To the best of our knowledge, this will be the first systematic review that aims to summarize studies on the relationship between motor performance and BP among children and adolescents; it will determine if there are any associations and, if so, how strong they are. In this study, the results from the cross-sectional studies can only provide information about association and not causality, whereas longitudinal studies can indicate the direction of the influences.

It is crucial to identify how motor performance and BP are associated in this age group, because BP and its harmful impacts increase during transition from childhood to adolescence. First, it is crucial to understand the risk factors and detect groups of frail children, who are particularly susceptible for developing BP [3] to prevent negative consequences. Second, because motor performance is trained in physical education classes, our result may provide valuable quantitative information to these educational and health professionals, which in turn could increase the quality of their physical education classes, avoiding unwanted BP.

Some limitations may be expected. First, various measurements and tools might lead to varying levels of motor performance outcomes. Second, the lack of clarity in the definition of BP might complicate the comparisons. Third, if no study meets our eligibility criteria, the review will be reported as an "empty review." However, even an "empty review" is relevant and might help stimulate appropriate future investigations [44].

Strengths of this study include assessment of a wide motor performance scope, absence of language and year restrictions, evaluation of quality and risk of bias analysis, and reporting of conflict of interest and ethical approval, all of which are often omitted from reviews. This systematic review will provide critical insights into the associations between motor performance and BP among children and adolescents that may be important for supporting the development of clinical practice guidelines and public health programs.

\section{Supplementary information}

Supplementary information accompanies this paper at https://doi.org/10. 1186/s13643-020-01468-6.

Additional file 1:. PRISMA-P (Preferred Reporting Items for Systematic Review and Meta-Analysis Protocols) 2015 checklist. Recommended items to address in a systematic review protocol.
Additional file 2:. Methodological quality assessment and strength of evidence.

\section{Abbreviations}

GRADE: Grading of Recommendations, Assessment, Development, and Evaluations; PRISMA: Preferred Reporting Items for Systematic Reviews and Meta-analyses; PROSPERO: International Prospective Register of Systematic Reviews; PECO: Population, Exposure, Comparator, and Outcome

\section{Acknowledgements}

The authors are grateful to the Department of Sports Science and Clinical Biomechanics (University of Southern Denmark) and the Instituto Federal Goiano for support. We also thank Anne-Marie Fiala Carlsen, librarian at UCL University College, for helping us build the search strategy.

\section{Authors' contributions}

MN, NW, and PK conceived the study idea. MN, NW, CM, and PK contributed to the design of the systematic review. MN, NW, CM, and PK contributed to the data analysis plan. All authors contributed to the write-up and editing of the manuscript and approved the final manuscript.

Funding

Not applicable.

Availability of data and materials

Not applicable.

Ethics approval and consent to participate

Not applicable.

Consent for publication

Not applicable.

Competing interests

The authors declare that they have no competing interests.

\section{Author details}

${ }^{1}$ Instituto Federal Goiano, Ceres, Brazil. ${ }^{2}$ Postgraduate Program in Health Sciences, Faculty of Medicine, Federal University of Goiás, Goiânia, Brazil. ${ }^{3}$ Department of Sports Science and Clinical Biomechanics, University of Southern Denmark, Campusvej 55, Odense, Denmark. ${ }^{4}$ Sports Medicine Clinic, Orthopedic Department, Hospital of Lillebaelt, Odense, Denmark. ${ }^{5}$ Health Sciences Research Centre, UCL University College, Odense, Denmark.

Received: 6 May 2020 Accepted: 26 August 2020

Published online: 14 September 2020

References

1. Beynon AM, Hebert JJ, Lebouef-Yde C, Walker BF. Potential risk factors and triggers for back pain in children and young adults. A scoping review, part II: unclear or mixed types of back pain. Chiropr Man Therap. 2019;27:61.

2. Beynon AM, Hebert JJ, Lebouef-Yde C, Walker BF. Potential risk factors and triggers for back pain in children and young adults. A scoping review, part I: incident and episodic back pain. Chiropr Man Therap. 2019;27:58.

3. Junge T, Wedderkopp N, Boyle E, Kjaer P. The natural course of low back pain from childhood to young adulthood - a systematic review. Chiropr Man Ther. 2019;27:1-10.

4. Kongsted A, Kent P, Axen I, Downie AS, Dunn KM. What have we learned from ten years of trajectory research in low back pain? BMC Musculoskelet Disord. 2016;17:220.

5. Buchbinder $R$, van Tulder $M$, Öberg B, et al. Low back pain: a call for action. Lancet. 2018;391:2384-8.

6. Foster NE, Anema JR, Cherkin D, et al. Prevention and treatment of low back pain: evidence, challenges, and promising directions. Lancet. 2018;391: 2368-83.

7. Twiddy H, Hanna J, Haynes L. Growing pains: understanding the needs of emerging adults with chronic pain. Br J Pain. 2017;11:108-18.

8. Noll M, Candotti CT, da Rosa BN, Vieira A, Loss JF. Back pain and its risk factors in Brazilian adolescents: a longitudinal study. Br J Pain. 2019;27: 204946371987175 
9. Joergensen AC, Hestbaek L, Andersen PK, Nybo Andersen AM. Epidemiology of spinal pain in children: a study within the Danish National Birth Cohort. Eur J Pediatr. 2019;178:695-706.

10. Dissing KB, Hestbæk L, Hartvigsen J, et al. Spinal pain in Danish school children - How often and how long? the CHAMPS Study-DK. BMC Musculoskelet Disord. 2017;18. https://doi.org/10.1186/s12891-017-1424-5.

11. Shah SA, Saller J. Evaluation and diagnosis of back pain in children and adolescents. J Am Acad Orthop Surg. 2016;24:37-45.

12. Kamper SJ, Henschke N, Hestbaek L, Dunn KM, Williams CM. Musculoskeletal pain in children and adolescents. 2016; 20: 275-84

13. MacDonald J, Stuart E, Rodenberg R. Musculoskeletal low back pain in school-aged children. JAMA Pediatr. 2017. https://doi.org/10.1001/ jamapediatrics.2016.3334.

14. Leboeuf-Yde C, Kyvik KO. At what age does low back pain become a common problem? A study of 29,424 individuals aged 12-41 years. Spine (Phila Pa 1976). 1998;23:228-34

15. Sundell C-G, Bergström E, Larsén K. Low back pain and associated disability in Swedish adolescents. Scand J Med Sci Sports. 2019;29:393-9.

16. Batley S, Aartun E, Boyle E, Hartvigsen J, Stern PJ, Hestbæk L. The association between psychological and social factors and spinal pain in adolescents. Eur J Pediatr. 2019;178:275-86.

17. Minghelli B. Musculoskeletal spine pain in adolescents: Epidemiology of non-specific neck and low back pain and risk factors. J Orthop Sci. 2019. https://doi.org/10.1016/j.jos.2019.10.008.

18. Andreucci A, Campbell P, Richardson E, Chen Y, Dunn KM. Sleep problems and psychological symptoms as predictors of musculoskeletal conditions in children and adolescents. Eur J Pain (United Kingdom) 2019; : 1-10.

19. Hebert JJ, Klakk H, Franz C, Sénéchal M, Manson N, Wedderkopp N. Spinal pain is prospectively associated with cardiovascular risk factors in girls but not boys (CHAMPS study-DK). Eur Spine J. 2019;28:2452-61.

20. Franz C, Møller NC, Korsholm L, Jespersen E, Hebert JJ, Wedderkopp N. Physical activity is prospectively associated with spinal pain in children (CHAMPS Study-DK). Sci Rep. 2017;7:4-11.

21. Noormohammadpour P, Rostami M, Mansournia MA, Farahbakhsh F, Pourgharib Shahi MH, Kordi R. Low back pain status of female university students in relation to different sport activities. Eur Spine J. 2015. https://doi. org/10.1007/s00586-015-4034-7

22. Noll M, Silveira EA, de Avelar IS. Evaluation of factors associated with severe and frequent back pain in high school athletes. PLoS One. 2017;12: e0171978.

23. Triki M, Koubaa A, Masmoudi L. Prevalence and risk factors of low back pain among undergraduate students of a sports and physical education institute in Tunisia. 2015; 1: 1-6.

24. Müller J, Müller S, Stoll J, Fröhlich K, Otto C, Mayer F. Back pain prevalence in adolescent athletes. Scand J Med Sci Sports. 2017;27:448-54.

25. R. K, T. H, K. W, et al. Gender differences in the prevalence of low back pain associated with sports activities in children and adolescents: a six-year annual survey of a birth cohort in Niigata City, Japan. BMC Musculoskelet Disord. 2019;20. https://doi.org/10.1186/s12891-019-2707-9.

26. Lubans DR, Morgan PJ, Cliff DP, Barnett LM, Okely AD. Fundamental movement skills in children and adolescents: review of associated health benefits. Sports Med. 2010;40:1019-35

27. Smith JJ, Eather N, Morgan PJ, Plotnikoff RC, Faigenbaum AD, Lubans DR. The health benefits of muscular fitness for children and adolescents: a systematic review and meta-analysis. Sports Med. 2014;44:1209-23.

28. García-Hermoso A, Ramírez-Campillo R, Izquierdo M. Is muscular fitness associated with future health benefits in children and adolescents? a systematic review and meta-analysis of longitudinal studies. Sports Med. 2019:49:1079-94.

29. Potthoff $T$, de Bruin ED, Rosser S, Humphreys BK, Wirth B. A systematic review on quantifiable physical risk factors for non-specific adolescent low back pain. J Pediatr Rehabil Med. 2018;11:79-94.

30. Larsen LR, Kristensen $P L$, Junge $T$, Rexen $C T$, Wedderkopp N. Motor performance as predictor of physical activity in children: the CHAMPS studyDK. Med Sci Sports Exerc. 2015;47:1849-56.

31. Larsen LR, Kristensen PL, Junge T, MØller SF, Juul-Kristensen B, Wedderkopp N. Motor performance as risk factor for lower extremity injuries in children. Med Sci Sports Exerc. 2016:48:1136-43.

32. Swain M, Henschke N, Kamper S, Gobina I, Ottová-Jordan V, Maher C. An international survey of pain in adolescents. BMC Public Health. 2014;14:447.
33. Moher D, Shamseer $L$, Clarke $M$, et al. Preferred reporting items for systematic review and meta-analysis protocols (PRISMA-P) 2015 statement. Syst Rev. 2015;4:1.

34. Morgan RL, Whaley $P$, Thayer KA, Schünemann HJ. Identifying the PECO: A framework for formulating good questions to explore the association of environmental and other exposures with health outcomes. Environ Int. 2018;121:1027-31.

35. Booth A, Clarke M, Dooley G, et al. The nuts and bolts of PROSPERO: an international prospective register of systematic reviews. Syst Rev. 2012;1:2.

36. Chui KC, Jorge M, Yen S-C, Lusardi MM. Orthotics and prosthetics in rehabilitation. Elsevier, 2020.

37. Ouzzani M, Hammady H, Fedorowicz Z, Elmagarmid A. Rayyan-a web and mobile app for systematic reviews. Syst Rev. 2016:5:210.

38. Stefani L, Galanti G, Padulo J, Bragazzi NL, Maffulli N. Sexual activity before sports competition: a systematic review. Front Physiol. 2016;7. https://doi. org/10.3389/fphys.2016.00246.

39. Downs $\mathrm{SH}$, Black N. The feasibility of creating a checklist for the assessment of the methodological quality both of randomised and non-randomised studies of health care interventions. J Epidemiol Community Health. 1998; $52 \cdot 377-84$.

40. Guyatt G, Oxman AD, Akl EA, et al. GRADE guidelines: 1. Introduction GRADE evidence profiles and summary of findings tables. J Clin Epidemiol. 2011;64:383-94.

41. Balshem $H$, Helfand $M$, Sch $H J$, et al. GRADE guidelines: 3. Rating the quality of evidence. 2011;64. https://doi.org/10.1016/j.jclinepi.2010.07.015.

42. Elia N, Von Elm E, Chatagner A, Pöpping DM, Tramèr MR. How do authors of systematic reviews deal with research malpractice and misconduct in original studies? A cross- sectional analysis of systematic reviews and survey of their authors; 2016. https://doi.org/10.1136/bmjopen-2015-010442.

43. Higgins J, Thomas J. Cochrane Handbook for Systematic Reviews of Interventions. Cochrane Collab. 2019. www.handbook.cochrane.org (accessed Sept 1, 2020).

44. Yaffe J, Montgomery P, Hopewell S, Shepard LD. Empty reviews: a description and consideration of Cochrane Systematic Reviews with no included studies. PLoS One. 2012;7:e36626.

\section{Publisher's Note}

Springer Nature remains neutral with regard to jurisdictional claims in published maps and institutional affiliations.

Ready to submit your research? Choose BMC and benefit from:

- fast, convenient online submission

- thorough peer review by experienced researchers in your field

- rapid publication on acceptance

- support for research data, including large and complex data types

- gold Open Access which fosters wider collaboration and increased citations

- maximum visibility for your research: over $100 \mathrm{M}$ website views per year

At BMC, research is always in progress.

Learn more biomedcentral.com/submissions 